\title{
SUCESSO REPRODUTIVO DE ESPÉCIES DISTÍLICAS DE Psychotria (RUBIACEAE) EM SUB-BOSQUE DE FLORESTA ATLÂNTICA ${ }^{1}$
}

\author{
Celice Alexandre Silva² e Milene Faria Vieira ${ }^{3}$
}

\begin{abstract}
RESUMO - As espécies distílicas Psychotria conjugens, P. hastisepala e P. sessilis (Rubiaceae) são típicas de sub-bosques sombreados. Ocorrem no maior fragmento de Floresta Estacional Semidecidual de Viçosa, Minas Gerais, Sudeste brasileiro - a Mata do Paraíso, com 194 ha. A distilia caracteriza-se pela presença dos morfos florais longistilos (L) e brevistilos (B) em indivíduos distintos e pela dependência de polinizações intermorfos (L x B ou B x L) para a produção de frutos; é esperada a proporção equilibrada (isopletia) dos indivíduos na população. Foram objetivos deste trabalho verificar, nas espécies citadas, a proporção dos morfos florais em uma área de 7 ha e a dependência por polinizadores, testando a incompatibilidade intramorfos ( $\mathrm{L} \times \mathrm{L}$ e B x B) por meio de polinizações manuais in vivo, a viabilidade dos grãos de pólen e dimorfismo dos grãos entre os morfos florais; e quantificar as produções de frutos e de sementes por morfo. Os morfos florais das espécies se encontram em proporções equilibradas. Houve incompatibilidade e a viabilidade dos grãos de pólen foi alta (>64\%). Verificou-se dimorfismo dos grãos, e os maiores diâmetros foram os de B. As produções de frutos e de sementes (uma ou duas) dos morfos de $P$. sessilis e de $P$. conjugens foram semelhantes e, em $P$. hastisepala, foram maiores em B. Na Mata do Paraíso, as condições adequadas, como o hábitat, a isopletia e a atuação de polinizadores, são fatores que parecem favorecer o sucesso reprodutivo e, consequentemente, a manutenção local das espécies estudadas.
\end{abstract}

Palavras-chave: Floresta Estacional Semidecidual, Frutificação, Sistema reprodutivo.

\section{REPRODUCTIVE SUCCESS OF DISTYLOUS SPECIES OF Psychotria (RUBIACEAE) OF UNDERSTORY ATLANTIC FOREST}

\begin{abstract}
The distylous species of Psychotria conjugens, P. hastisepala e P. sessilis (Rubiaceae) are typical of understory shady. It occurs in the largest fragment (194 ha) of semideciduous forest in Viçosa, Minas Gerais State, southeastern Brazil, where the present study was carried out. The distyly is characterized by the presence of pin $(L)$ and thrum $(B)$ floral morphs in different individual and the dependence on intermorph pollinations ( $L \times B$ or $B \times L$ ) of fruit set, is expected to be at balanced proportions (isopleths) of the individuals in the population. The purpose of this study was to determine, for the species mentioned above, the proportion offloral morphs in an area of seven hectares, the dependence on pollinators by testing the intramorph incompatibility ( $L x L$ and $B \times B$ ) by in vivo controlled pollinations; the viability of pollen grains and the existence of grain size dimorphism among floral morphs, and to quantify the production of fruits and seeds per morph. The floral morphs of the species was found in balanced proportions. There was an incompatibility and the viability of pollen grains was high (> 64\%). Grains presented dimorphism; the highest diameters were the B. The production of fruits and seeds (one or two) of the morphs of $\boldsymbol{P}$. sessilis and $\boldsymbol{P}$. conjugens were similar and in $\mathrm{P}$. hastisepala they were higher in B. In the study area, at the correct conditions, such as habitat, isopleths and the role of pollinator are the factors that seem to promote the reproductive success and therefore the maintenance of local species.
\end{abstract}

Keywords: Breeding system, Fruit set, Semideciduous forest.

\footnotetext{
${ }^{1}$ Recebido em 05.03.2011 aceito para publicação em 05.04.2013.

${ }^{2}$ Universidade do Estado de Mato Grosso, Laboratório de Botânica, Centro de Estudos, Pesquisas e Desenvolvimento Agro ambiental (CPEDA). E-mail:<celicealexandre645@ hotmail.com>.

${ }^{3}$ Universidade Federal de Viçosa, Centro de Ciências Biológicas e da Saúde, Departamento de Biologia Vegetal. E-mail:<mfvieira@ufv.br>.
} 


\section{INTRODUÇÃO}

Psychotria é o gênero de Rubiaceae que possui o maior número de espécies distílicas (ALMEIDAe ALVES, 2000), encontradas, comumente, em sub-bosques de florestas tropicais (HAMILTON, 1990; TAYLOR, 1996). Nesse estrato florestal exercem importante papel ecológico, incluindo o fornecimento de recursos florais aos polinizadores - abelhas (RAMOS; SANTOS, 2006; LOPES; BUZATO, 2007), borboletas (LOPES e BUZATO, 2007) e beija-flores (CASTRO; ARAUJO, 2004; COELHO; BARBOSA, 2004; LOPES; BUZATO, 2007) - e de frutos aos dispersores como pássaros (ALMEIDA et al., 2006).

A distilia é um dimorfismo floral controlado geneticamente e caracterizado, principalmente, pelo posicionamento recíproco de estigmas e anteras entre dois morfos florais, brevistilo (B) e longistilo (L), produzidos em indivíduos distintos e na presença de um sistema de incompatibilidade após polinizações ilegítimas (auto e intramorfos). Nas espécies distílicas, a frutificação, geralmente, é dependente de polinizações legítimas (intermorfos) realizadas pelos polinizadores (BARRETT, 1992).

O sucesso reprodutivo sensu (CONTRERAS; ORNELAS, 1999) de espécies distílicas é dependente de vários fatores associados (SILVA et al., 2010), entre eles o dimorfismo floral (ORNELAS et al., 2004ab; TEIXEIRA; MACHADO, 2004a; PEREIRA et al., 2006b), a isopletia, ou seja, a proporção equilibrada entre os morfos na população (SOBREVILLA et al., 1983; PAILLER; THOMPSON, 1997; KOCH et al., 2010) e a atuação efetiva de polinizadores (LOPES; BUZATO, 2007; SILVA et al., 2010).

Em fragmentos florestais, comuns nos dias atuais, em especial na Floresta Atlântica, algumas espécies distílicas encontram-se em proporção desequilibrada (anisopletia) (COELHO;BARBOSA, 2003; CONSOLARO et al., 2009) em razão, provavelmente, da fragmentação, que resulta em perda de hábitat e de polinizadores. Espécies distílicas anisopléticas tendem à autocompatibilidade (GANDERS, 1979; ROSSI et al., 2005) e reproduzem-se, principalmente, por propagação vegetativa (SOBREVILA et al., 1983; ROSSI et al., 2005), pois as suas frutificações são baixas (ROSSI et al., 2005; KOCH et al., 2010).

Na Mata do Paraíso, maior fragmento de Floresta Estacional Semidecidual do Município de Viçosa, Zona da Mata mineira e local deste estudo, foram identificadas
30 espécies de Rubiaceae, 16 delas distílicas e restritas ao sub-bosque. Entre as distílicas, 10 pertencem ao gênero Psychotria, incluindo P. conjugens Müll. Arg., P. hastisepala Müll. Arg. e P. sessilis Vell., que são as mais comumente observadas (PEREIRA et al., 2006a). De acordo com Pereira et al. (2006b), essas espécies, após polinizações manuais in vitro, mostram-se autoincompatíveis e possuem dimorfismos florais, além do posicionamento recíproco de estigmas e anteras, e, com exceção de P. conjugens, apresentam proporção equilibrada dos morfos na população. Entretanto, essas autoras não realizaram polinizações manuais in vivo e nem verificaram a viabilidade dos grãos de pólen e a frutificação natural dessas plantas, que são informações essenciais para o entendimento do seu sucesso reprodutivo.

Considerando a riqueza de espécies distílicas de Rubiaceae na Mata do Paraíso, em especial de Psychotria, e a sua importância ecológica, foi objetivo buscar informações adicionais sobre a reprodução sexuada de $P$. conjugens, $P$. hastisepala e $P$. sessilis. Para isso, verificaram-se a proporção dos morfos florais em uma área de 7 ha e a dependência por polinizadores, testando a incompatibilidade intramorfos por meio de polinizações manuais in vivo, a viabilidade dos grãos de pólen e dimorfismo dos grãos (tamanho) entre os morfos florais; e quantificaram-se as produções de frutos e de sementes por morfo.

\section{MATERIAL E MÉTODOS}

\section{1. Área de estudo e espécies estudadas}

O estudo foi desenvolvido na Mata do Paraíso, o maior fragmento florestal do Município de Viçosa ( $20^{\circ} 45^{\prime} \mathrm{S}$ e $\left.42^{\circ} 51^{\prime} \mathrm{W}\right)$, com cerca de 194 ha, no período de setembro de 2004 a abril de 2005 e de setembro de 2005 a abril de 2006. A Mata do Paraíso é uma área de preservação ambiental que apresenta pequenas porções de brejo com taboa (Typha sp.), pastagem abandonada ocupada por capim-gordura (Melinis minutiflora $\mathrm{P}$. Beauv.) e um pequeno açude, além da vegetação florestal encontrada nas encostas e nos topos de morros (RIBON, 2005). A Mata do Paraíso está sob a supervisão do Departamento de Engenharia Florestal da Universidade Federal de Viçosa. Sua vegetação original está inserida nos domínios da Floresta Atlântica (RIZZINI, 1992) e foi classificada por Veloso et al. (1991) como Floresta Estacional Semidecidual Montana. 
Viçosa encontra-se a $650 \mathrm{~m}$ de altitude, e o seu clima é caracterizado por apresentar temperatura média anual de $19^{\circ} \mathrm{C}$, precipitação anual de 1.300 a $1.400 \mathrm{~mm}$, com o período de maior precipitação entre outubro e março e umidade relativa do ar de 80 a $85 \%$. Durante o período de estudo, observou-se que a maior média diária de precipitação ( $\bar{X}=5,22 \mathrm{~mm}$ ) concentrou-se nos meses de dezembro a março, período em que também foram observadas as temperaturas mais elevadas $(\bar{X}$ $=21,44^{\circ} \mathrm{C}$ ). Os dados climatológicos foram fornecidos pela Estação Meteorológica da Universidade Federal de Viçosa.

Na Mata do Paraíso, a família Rubiaceae está amplamente representada desde ervas, arbustos e árvores (HIGUCHI et al., 2006). Psychotria conjugens e $P$. hastisepala são subarbustos de 1,0 a $2,5 \mathrm{~m}$, ambas com distribuição restrita ao território nacional, ocorrendo do Sul do Pará até o Rio Grande do Sul (ANDERSSON, 1992). P. sessilis é arbusto de 1,5 a 3,5 $\mathrm{m}$ de altura e, além do Brasil, ocorre em vários outros países sulamericanos (ANDERSSON, 1992). Todas as três espécies habitam o sub-bosque, florescem na estação chuvosa e suas flores apresentam antese diurna, corola tubulosa, de cor branca ou creme e têm duração de um dia (PEREIRA et al., 2006b).

Os frutos são drupáceos e ornitocóricos e cada fruto produz de uma a duas sementes (observação pessoal). Espécimes testemunhas encontram-se depositados no Herbário VIC da Universidade Federal de Viçosa ( ${ }^{\circ}$ s. 26.963, 26.964 e 26.974).

\subsection{Proporção entre os morfos florais}

Em uma área de 7 ha foi feita a contagem e identificados os morfos de todos os indivíduos floridos de cada espécie. A distância mínima mantida entre indivíduos foi de $3 \mathrm{~m}$. Desse modo, tentou-se evitar, na contagem, a inclusão dos clones produzidos pelas espécies por meio de reprodução vegetativa.

\subsection{Tamanho e viabilidade dos grãos de pólen}

Para as medições foram coletados cinco botões, em pré-antese, de cinco indivíduos de cada morfo e estocados em ácido acético. As anteras, de cada botão floral, foram maceradas em lâmina e coradas com carmim acético (RADFORD et al., 1974). Em cada lâmina foram medidos oito grãos, totalizando 200 grãos por morfo. Devido ao seu formato circular, em cada grão foram realizadas seis medidas (diâmetros) e tirada a média. Imagens dos grãos de pólen foram capturadas em microscópio óptico binocular (OLYMPUS AX 70) e as medidas $(\mu \mathrm{m})$, obtidas usando-se o software Imagempro plus.

Para determinação da viabilidade dos grãos de pólen, foram coletados outros cinco botões em pré-antese, de cinco indivíduos diferentes, totalizando 25 botões por morfo floral por espécie. Esses botões foram estocados em etanol $70 \%$. As anteras, de cada botão, foram maceradas em lâmina, sobre uma gota de carmim acético (RADFORD et al., 1974). Em cada lâmina (total de 50) foram contados até 200 grãos (KEARNS; INOUYE, 1993).

\subsection{Polinizações manuais in vivo}

Os tratamentos foram realizados em duas estações reprodutivas e consecutivas, exceto no de $P$. conjugens. Nesta espécie, as polinizações manuais foram realizadas na floração de 2005/2006. Utilizou-se a metodologia de Bawa e Beach (1983): B x B e L x L (autopolinização e polinização intramorfo) e B x L e L x B (polinizações intermorfos). Em todas as espécies, as inflorescências foram previamente isoladas com sacos de tecido do tipo organza, e as flores polinizadas tiveram seus pedicelos marcados com linhas coloridas (cada cor correspondendo a um tipo de polinização). As polinizações manuais foram realizadas no período da manhã (das 7 às 9 h). Após os tratamentos, as inflorescências permaneceram ensacadas até a frutificação ou a queda da flor.

Algumas inflorescências foram marcadas para avaliar a eficiência da polinização natural (controle). A porcentagem da polinização natural foi calculada, considerando-se o número total de flores e de frutos em desenvolvimento por inflorescência.

\subsection{Produção de frutos e sementes}

Em cinco inflorescências de 10 indivíduos de cada morfo de cada espécie estudada, foi feita a contagem do número de frutos/inflorescência oriundos de polinizações naturais e de sementes/fruto.

\subsection{Análise estatística}

Análises estatísticas foram realizadas usando o Programa "General Linear Model", no software Statistica versão 5.5 (STATSOFT, 2000). Nas análises de variância foi aplicado o teste One-way ANOVA (ZAR, 1999). 


\section{RESULTADOS}

\subsection{Proporção de morfos}

A proporção de indivíduos foi de 18B:15L em $P$. conjugens $\left(\chi^{2}=1,45 ; \mathrm{P}=0,23\right), 28 \mathrm{~B}: 26 \mathrm{Lem}$ P. hastisepala $\left(\chi^{2}=0,66 ; \mathrm{P}=0,41\right)$ e 93B:87L em $P$. sessilis $\left(\chi^{2}=2,18\right.$; $\mathrm{P}=0,14)$. Essas proporções não diferem de 1:1 e, por isso, a razão entre os morfos é equilibrada, ou seja, há isopletia.

\subsection{Viabilidade e tamanho dos grãos de pólen}

A viabilidade dos grãos de pólen encontra-se na Tabela 1; não houve diferença entre os morfos de cada espécie. Os diâmetros dos grãos de pólen das flores brevistilas, em todas as espécies, foram significativamente maiores que os das longistilas (Tabela 1).

\subsection{Sistema reprodutivo}

As espécies apresentaram incompatibilidade após polinizações ilegítimas (Tabela 2). Os frutos observados em $P$. conjugens e P. hastisepala, oriundos da autopolinização L x L, devem ser resultantes de contaminação acidental de pólen do outro morfo.

As frutificações obtidas nas polinizações naturais (controle, Tabela 2) indicam a atuação de polinizadores. Entretanto, a atuação parece ser limitada se essas frutificações forem comparadas com aquelas obtidas nas polinizações legítimas, principalmente em P. conjugens.

Tabela 1 - Viabilidade e tamanho de grãos de pólen de Psychotria sessilis, $P$. conjugens e $P$. hastisepala na Mata do Paraíso, Viçosa, Minas Gerais.

Table 1 - Viability and size of pollen grains of Psychotria sessilis, P. conjugens and P. hastisepala in the Mata do Paraiso, Viçosa, Minas Gerais.

\begin{tabular}{|c|c|c|c|c|c|c|}
\hline \multirow{2}{*}{ Espécie/morfos } & \multicolumn{3}{|c|}{ Viabilidade de pólen (\%) } & \multicolumn{3}{|c|}{ Diâmetro de pólen $(\mu \mathrm{m})$} \\
\hline & $\bar{X}$ & $\mathrm{DP}$ & $\mathrm{F}$ & $\bar{X}$ & DP & $\mathrm{F}$ \\
\hline \multicolumn{7}{|l|}{ P. sessilis/ } \\
\hline B & \multicolumn{2}{|c|}{$81,62 \pm 14,23$} & \multirow{2}{*}{0,49 (NS) } & 8,40 & $\pm 0,53$ & \multirow{2}{*}{$797,1 * *$} \\
\hline $\mathrm{L}$ & 76,16 & 28,19 & & $7,1 \mathrm{C}$ & $\pm 0,31$ & \\
\hline \multicolumn{7}{|l|}{ P. conjugens/ } \\
\hline B & \multicolumn{2}{|c|}{$69,34 \pm 26,06$} & \multirow[t]{2}{*}{$2,44(\mathrm{NS})$} & 8,40 & $\pm 0,36$ & \multirow[t]{2}{*}{$487,4 * *$} \\
\hline $\mathrm{L}$ & 64,40 & 25,54 & & 7,72 & $\pm 0,24$ & \\
\hline \multicolumn{7}{|l|}{ P. hastisepalal } \\
\hline B & \multicolumn{2}{|c|}{$90,82 \pm 7,51$} & 0,11 (NS) & \multicolumn{2}{|c|}{$9,77 \pm 0,32$} & $738,4 * *$ \\
\hline $\mathrm{L}$ & 90,71 & 9,29 & & 8,7 & $\pm 0,42$ & \\
\hline
\end{tabular}

${ }^{* *} \mathrm{P}<0,05$ pelo teste One-Way Anova.

\subsection{Produção de frutos e sementes}

O número de frutos e sementes de cada morfo floral das espécies estudadas encontra-se na Tabela 3. Não houve diferença no número de frutos entre os morfos de $P$. sessilis, na porcentagem de frutos com uma e duas sementes e no número total de sementes produzidas. $P$. conjugens também não apresentou diferenças nesses mesmos parâmetros. Entretanto, as flores brevistilas de $P$. hastisepala produziram maior número de frutos do que as flores longistilas (Tabela 3), assim como foi maior o número de frutos com duas sementes e de sementes totais produzidas; não houve diferença entre morfos, apenas na porcentagem de frutos produzidos com uma semente.

\section{DISCUSSÃO}

A isopletia verificada em $P$. sessilis e $P$. hastisepala confirmaram os dados de Pereira et al. (2006b). Essas autoras, entretanto, mencionaram que $P$. conjugens, na Mata do Paraíso, é anisoplética, diferindo dos resultados deste estudo. Essa diferença deve ser resultante do tamanho da área amostrada; a área deste estudo é cerca de sete vezes maior que a utilizada por Pereira et al. (2006b). A razão equilibrada entre os morfos florais é característica desejável em espécies distílicas (MURCIA, 1996), pois, desse modo, podem contribuir igualmente para o seu sucesso reprodutivo e tem sido registrada em outras espécies distílicas de Psychotria (CASTRO; ARAÚJO, 2004; COELHO; BARBOSA, 2004; ROSSI et al., 2005; KOCH et al., 2010).

A alta viabilidade dos grãos de pólen, tal como verificado, é característica comum em espécies heterostílicas (DULBERGER, 1992), também registrada em Psychotria poeppigiana (COELHO; BARBOSA, 2004), P. barbiflora (TEIXEIRA e MACHADO, 2004a) e P. carthagenenis (KOCH et al., 2010). O maior diâmetro do pólen em flores brevistilas também é resultado esperado em espécies distílicas, inclusive em espécies de Psychotria (JUNGMENDAÇOLLI; MELHEM, 1995). Tais diferenças morfométricas, segundo Ganders (1979), são necessárias para que haja maior armazenamento de reservas nos grãos das flores brevistilas. Essas reservas adicionais dariam condições de crescimento dos seus tubos polínicos nos longos estiletes das flores longistilas.

Entre as espécies estudadas, o maior sucesso reprodutivo foi registrado em $P$. sessilis, favorecido pelas altas frutificações naturais, viabilidades dos grãos 
Tabela 2 - Resultados das polinizações manuais, in vivo, em flores de espécies de Psychotria, na Mata do Paraíso, Viçosa, Minas Gerais. AP = Autopolinização, PI = Polinização intramorfos, $\mathrm{PL}=$ Polinização legitima, $\mathrm{CO}=\mathrm{Controle}$, $\mathrm{B}=$ Morfo brevistilo, $\mathrm{L}=$ Morfo longistilo.

Table 2 - Results of pollination, in vivo, in flowers of species of Psychotria in the Mata do Paraiso, Viçosa, Minas Gerais. $A P=$ Self-pollination, $P I=$ pollination intramorph, $P L=$ Legitimate pollination, $C O=C o n t r o l, B=$ Thrum morph, $L=$ Pin morph.

\begin{tabular}{|c|c|c|c|c|c|c|c|c|c|c|}
\hline \multirow{2}{*}{ Testes } & \multirow{2}{*}{ Morfos } & \multicolumn{3}{|c|}{ P. sessilis } & \multicolumn{3}{|c|}{ P. conjugens } & \multicolumn{3}{|c|}{ P. hastisepala } \\
\hline & & In.d $\mathrm{N}^{\circ}$ & Flor $\mathrm{N}^{\circ}$ & Fruto $\mathrm{N}^{\circ} \%$ & In.d N $\mathrm{N}^{\mathrm{O}}$ & Flor $\mathrm{N}^{\circ}$ & Fruto $\mathrm{N}^{\circ} \%$ & In.d $\mathrm{N}^{\mathrm{o}}$ & Flor $\mathrm{N}^{\circ}$ & Fruto $\mathrm{N}^{\circ} / \%$ \\
\hline \multirow[t]{2}{*}{ AP } & $\mathrm{B} \times \mathrm{B}$ & 10 & 18 & $0 / 0$ & 5 & 8 & $2 / 25$ & 11 & 16 & $0 / 0$ \\
\hline & $\mathrm{LxL}$ & 10 & 22 & $0 / 0$ & 8 & 10 & $1 / 10$ & 13 & 15 & $1 / 6$ \\
\hline \multirow[t]{2}{*}{ PI } & $\mathrm{B} \times \mathrm{B}$ & 9 & 12 & $0 / 0$ & 6 & 8 & $0 / 0$ & 12 & 15 & $0 / 0$ \\
\hline & $\mathrm{LxL}$ & 12 & 15 & $0 / 0$ & 6 & 8 & $0 / 0$ & 10 & 15 & $0 / 0$ \\
\hline \multirow[t]{2}{*}{ PL } & $\mathrm{BxL}$ & 15 & 18 & $13 / 72$ & 6 & 7 & $6 / 86$ & 10 & 13 & $4 / 31$ \\
\hline & $\mathrm{LxB}$ & 12 & 15 & $11 / 73$ & 8 & 9 & $6 / 66$ & 9 & 16 & $6 / 37$ \\
\hline \multirow[t]{2}{*}{$\mathrm{CO}$} & B & 8 & 72 & $38 / 53$ & 5 & 35 & $10 / 29$ & 8 & 55 & $32 / 58$ \\
\hline & $\mathrm{L}$ & 9 & 63 & $45 / 72$ & 5 & 38 & $8 / 21$ & 9 & 81 & $16 / 20$ \\
\hline
\end{tabular}

Tabela 3 - Número de frutos, porcentagem de frutos com uma ou duas sementes e total de sementes produzidas, nos morfos florais de espécies de Psychotria, na Mata do Paraíso, Viçosa, Minas Gerais.

Table 3 - Fruit number, percentage of fruit with one or two seeds and total seeds produced in the floral morphs of Psychotria species in the Mata do Paraiso, Viçosa, Minas Gerais.

\begin{tabular}{|c|c|c|c|c|c|c|c|c|c|}
\hline \multirow{2}{*}{ Espécie } & \multirow{2}{*}{ Morfo } & \multicolumn{2}{|r|}{ Frutos } & \multicolumn{4}{|c|}{ Frutos $(\%)$ com } & \multicolumn{2}{|c|}{ Total de sementes } \\
\hline & & $\left(\mathrm{N}^{\circ}\right)$ & & 1 semente & & 2 sementes & & $\left(\mathrm{N}^{\circ}\right)$ & \\
\hline \multirow[t]{2}{*}{ P. sessilis } & B & 569 & & 27,60 & & 72,40 & & 981 & \\
\hline & $\mathrm{L}$ & 717 & $\mathrm{~F}=0,32 ; \mathrm{P}=0,56$ & 26,10 & $\mathrm{~F}=0,09 ; \mathrm{P}=0,76$ & 73,90 & $\mathrm{~F}=0,43 ; \mathrm{P}=0,51$ & 1248 & $\mathrm{~F}=0,89 ; \mathrm{P}=0,34$ \\
\hline \multirow[t]{2}{*}{ P. conjugens } & $\mathrm{B}$ & 196 & & 26,50 & & 73,50 & & 340 & \\
\hline & $\mathrm{L}$ & 203 & $\mathrm{~F}=0,009 ; \mathrm{P}=0,92$ & 44,30 & $\mathrm{~F}=2,03 ; \mathrm{P}=0,17$ & 55,70 & $\mathrm{~F}=0,30 ; \mathrm{P}=0,58$ & 316 & $\mathrm{~F}=0,08 ; \mathrm{P}=0,77$ \\
\hline \multirow[t]{2}{*}{ P. hastisepala } & B & 229 & & 6,10 & & 93,90 & & 444 & \\
\hline & $\mathrm{L}$ & 121 & $\mathrm{~F}=4,80 ; \mathrm{P}=0,04$ & 10,80 & $\mathrm{~F}=0,02 ; \mathrm{P}=0,88$ & 89,20 & $\mathrm{~F}=6,03 ; \mathrm{P}=0,02$ & 229 & $F=6,61 ; P=0,013$ \\
\hline
\end{tabular}

de pólen e produções de frutos e sementes nos morfos, além da isopletia. É amplamente visitada pelos polinizadores, e sua floração é abundante (SILVA, 2007). Na área de estudo, essa espécie é a mais amplamente distribuída; foram amostrados, no mínimo, 3,4 vezes mais indivíduos de $P$. sessilis em relação às demais espécies (180 vs 33 de P. conjugens e 54 de P. hastisepala). Todas essas características parecem agir em sinergia, garantindo o seu sucesso reprodutivo. Em espécies nas quais a polinização cruzada é obrigatória e realizada por insetos, tal como em $P$. sessilis, o tamanho da população está diretamente relacionado com a atração de polinizadores e tem influência positiva no seu sucesso reprodutivo(SIH e BALTUS, 1987; AGUILAR; GALETO, 2004).
Psychotria conjugens apresentou frutificações naturais, viabilidades dos grãos de pólen e produções de frutos e sementes menores que as obtidas em $P$. sessilis, assim como foram menores as intensidades de sua floração e de visitação (SILVA, 2007). Essa espécie foi a que apresentou o menor número de indivíduos amostrados, embora esteja em isopletia. Segundo Sih e Baltus (1987), pequenas populações têm sua atratividade reduzida, e o seu sucesso reprodutivo é afetado. De fato, entre as espécies estudadas, $P$. conjugens foi a que apresentou a menor quantidade total de sementes, exceto em relação ao morfo longistilo de $P$. hastisepala, e as menores porcentagens de frutos com duas sementes. Esses resultados indicam que há limitação de fluxo de pólen na população, semelhante ao registrado em 
outras espécies autoincompatíveis e com populações reduzidas (JENNERSTEN, 1988; AGREN, 1996). A quantidade de pólen depositado no estigma tem sido fator inibidor no sucesso reprodutivo de espécies autoincompatíveis (TOMIMATSU; OHARA, 2002). Verifica-se, portanto, que a razão equilibrada entre os morfos de $P$. conjugens, por si, não garantiu frutificações abundantes e demonstrou que a isopletia não deve ser vista como fator isolado que garanta o sucesso reprodutivo de espécies distílicas na população.

As flores longistilas de $P$. conjugens apresentaram tendência de ser mais visitadas que as brevistilas (SILVA, 2007) e, apesar disso, produziram menor quantidade total de sementes e maior porcentagem de frutos com uma semente. Esses resultados indicam que, nessas flores, a exposição do estigma, fora do tubo da corola, pode ter favorecido a deposição de pólen incompatível, uma vez que os polinizadores tendem a visitar sequencialmente todas as flores do mesmo indivíduo, geitonogamia sensu (RICHARDS, 1997). Esse comportamento poderia causar a oclusão do estigma e estilete com grãos de pólen incompatíveis e impossibilitaria ou diminuiria as chances de germinação de grãos de pólen compatíveis, tal como verificado por Ganders (1979), Dulberger (1992) e Dulberger e Ornduff (2000) em outras espécies distílicas.

Psychotria hastisepala foi, entre as espécies estudadas, a que apresentou diferenças entre os morfos, em relação às frutificações naturais e à produção de frutos e sementes. As flores brevistilas produziram duas vezes mais frutos que as longistilas, no controle, e cerca de duas vezes mais sementes. Essa espécie também foi a única, entre as estudadas, que teve reprodução vegetativa (observação pessoal). Esse mecanismo reprodutivo pode alterar a proporção esperada em espécies heterostílicas, privilegiando o morfo que possui maior capacidade de instalação de novos clones (GRANDISIOLI, 1997). Entretanto, isso não parece ocorrer com $P$. hastisepala, dada a sua isopletia, na área de estudo. Portanto, outros fatores têm afetado o sucesso reprodutivo das flores longistilas.

P. hastisepala foi também, entre as espécies estudas, a que apresentou a menor intensidade de floração, número de flores abertas/indivíduo/dia e frequência de visitação. Os polinizadores, observados por dois anos consecutivos, foram inconstantes, tanto entre os morfos quanto entre os anos estudados (SILVA, 2007). No entanto, as flores brevistilas não pareceram ter sido afetadas, considerando o seu maior sucesso reprodutivo em relação às longistilas e aos morfos florais de $P$. conjugens.

Sucessos distintos entre morfos de espécies distílicas têm sido registrados, e as justificativas para essas diferenças envolveram o comportamento de polinizadores (STONE, 1996; TEIXEIRA; MACHADO, 2004ab; CASTRO; ARAÚJO, 2004) e a oclusão do estigma de flores longistilas com pólen incompatível (TEIXEIRA; MACHADO, 2004b). Entretanto, estudos realizados com $P$. barbiflora demonstraram que o sucesso reprodutivo dos morfos, quando analisados em populações distintas, pode variar. Monteiro et al. (1991) observaram que as flores longistilas de P. barbiflora produziram maior número de frutos que as brevistilas em população de Cerrado, enquanto Teixeira e Machado (2004a) analisando a mesma espécie, em uma população de Mata Atlântica, verificaram maior produção de frutos nas brevistilas. O baixo sucesso das longistilas em $P$. hastisepala, na área deste estudo, também pode estar relacionado à oclusão do estigma, como também verificado por Webb e Lloyd (1986) em outras espécies distílicas. Além disso, outro fator que pode ter afetado o sucesso dessas flores foram os danos (herbivoria) causados por coleópteros, frequentemente registrados na área de estudo, aos estigmas expostos do morfo longistilo (observação pessoal). Esses danos, entretanto, não foram quantificados, e estudos posteriores poderão esclarecer melhor seu significado.

Os resultados indicaram que, embora a Mata do Paraíso seja um fragmento florestal em regeneração por cerca de 45 anos (RIBON, 2005), as condições ambientais já estabelecidas têm favorecido a permanência das espécies estudadas de Psychotria.

\section{CONCLUSÕES}

A proporção de indivíduos das espécies distílicas estudadas é equilibrada (isopletia): 93B:87L em Psychotria sessilis, 28B:26Lem P. hastisepala e 18B:15L em $P$. conjugens.

As espécies são autoincompatíveis e apresentam grãos de pólen com alta viabilidade; os maiores diâmetros dos grãos são os das flores brevistilas.

A produção de frutos e sementes entre os morfos de $P$. sessilis e de $P$. conjugens foi semelhante e a de $P$. hastisepala, desigual, sendo, nesse caso, maior no brevistilo. 
O maior sucesso reprodutivo foi de $P$. sessilis, favorecido pelas altas produções de frutos e de sementes, além da alta viabilidade dos grãos, da isopletia e do grande número de indivíduos amostrados. Nas demais espécies, as produções de frutos e de sementes foram menores, mas ainda sim mostram que estavam em equilíbrio na área de estudo.

\section{AGRADECIMENTOS}

Ao CNPq, pela bolsa de PQ concedida à segunda autora; à FAPEMIG, pelo apoio financeiro (Universal CRA1868/2005); e ao Departamento de Engenharia Florestal, por permitir o acesso às plantas na Mata do Paraíso. Este estudo é parte da Tese de Doutorado da primeira autora.

\section{REFERÊNCIAS}

AGREN, J. Population size, pollinator limitation, and seed set in the self-incompatible herb Lytrum salicaria. Ecology, v.77, n.3, p.1799-1990, 1996.

AGUILAR, R.; GALETTO, L. Effects of forest fragmentation on male and female reproductive sucess in Cestrum parqui (Solanaceae).

Oecologia, v.138, n.4, p.513-520, 2004.

ALMEIDA, E. M.; ALVES, M. A. Fenologia de Psychotria nuda e P. brasilensis (Rubiaceae) em uma área de Floresta Atlântica no sudeste do Brasil. Acta Botânica Brasílica, v.14, n.3, p.335-346, 2000.

ALMEIDA, E. M. et al. Potential bird dispersers of Psychotria in a area of Atlantic Forest on Ilha Grande, RJ, southeastern Brazil: A biochemical analysis of the fruits. Brazilian Journal of Biology, v.66, n.1A, p.1-8, 2006.

ANDERSSON, L. A. A provisional checklist of Neotropical Rubiaceae. Scripta Botanica Belgica, v.1, n.1, p.1-119, 1992.

BARRETT, S. C. H. Heterostylous genetic polymorphisms: model system for evolutionary analysis. In: BARRETT, S. C. H. (Ed.).

Evolution and function of heterostyly. New York: Springer, 1992. p.1-24.

BAWA, K. S.; BEACH, J. H. Self- incompatibility systems in the Rubiaceae of a tropical lowland wet forest. American Journal of Botany, v.70, n.6, p.1281-1288,1983.
CASTRO, C. C.; ARAúJO, A. C. Distyly and sequential pollinators of Psychotria nuda (Rubiaceae) in the Atlantic rain forest, Brazil. Plant Systematics and Evolution, v.244, n.1, p.131-139, 2004.

COELHO, C. P.; BARBOSA, A. A. A. Biologia reprodutiva de Palicourea macrobotrys Ruiz \& Pavon (Rubiaceae): um possivel caso de homostilia no genero Palicourea Aubl. Revista Brasileira de Botânica, v.26, n.3, p.403-413, 2003.

COELHO, C. P.; BARBOSA, A. A.A. Biologia reprodutiva de Psychotria poeppigiana Mull. Arg. (Rubiaceae) em mata de galeria. Acta Botânica Brasílica, v.18, n.3, p.481-489, 2004.

CONSOLARO, H. et al. Distilia e homostilia em especies de Palicourea Aubl. (Rubiaceae) do Cerrado do Brasil Central. Revista Brasileira de Botânica, v.32, n.4, p.655-667, 2009.

CONTRERAS, P. S.; ORNELAS, J. F.

Reproductive conflicts of Palicourea padifolia (Rubiaceae) a distylous shrub of a Tropical cloud forest in Mexico. Plant Systematics and Evolution, v.219, n.1, p.225-241, 1999.

DULBERGER, R. Floral polymorphisms and their functional significance in the heterostylous syndrome. In: BARRETT, S. C. H. (Ed.).

Evolution and function of heterostyly. New York: Springer, 1992. p.40-48.

DULBERGER, R.; ORNDUFF, R. Stigma morphology in distylous and non-heterostylous species of Villarsia (Menyanthaceae). Plant Systematics and Evolution v.225, n.1, p.171-184, 2000.

GANDERS, F. R. The biology of heterostyly. New Zealand Journal of Botany, v.17, n.1, p.607-635, 1979.

GRANDISOLI, E. A. C. Biologia reprodutiva e estrutura da população de Psychotria suterella Muell. Arg. (Rubiaceae) em um fragmento de mata secundaria em São Paulo (SP). 1997. 96f. Dissertação (Mestrado em Botânica) - Universidade de São Paulo - USP, São Paulo, 1997.

Revista Árvore, Viçosa-MG, v.37, n.2, p.289-297, 2013 
HAMILTON, C. W. Variation on a distylous theme in Mesoamerican Psychotria subgenus Psychotria (Rubiaceae). Memoirs of the New York Botanical Garden, v.55, n.1, p.62-75, 1990.

HIGUCHI, P. et al. Composição florística da regeneração natural de espécies arbóreas ao longo de oito anos em um fragmento de Floresta Estacional Semidecidual em Viçosa-MG. Revista Árvore, v.30, n.6, p.893-904, 2006.

JENNERSTEN, O. Pollination in Dianthus deltoides (Caryophyllaceae): effects of habitat fragmentation on visitation and seed set. Conservation Biology, v.2, n.2, p.359-366, 1988.

JUNG-MENDAÇOLLI, S. L.; MELHEM, T. S. Grãos de pólen de espécies heterostílicas de Rubiaceae. Revista Brasileira de Botânica, v.18, n.1, p.61-93, 1995.

KEARNS, C. A.; INOUYE, D. W. Techniques for pollination biologists. Boulder: University Press of Colorado, 1993. 583p.

KOCH, A. K.; SILVA, P. C.; SILVA, C. A. Biologia reprodutiva de Psychotria carthagenensis (Rubiaceae), espécie distílica de fragmento florestal de mata ciliar, centro-oeste do Brasil. Rodriguésia, v.61, n.3, p.551-558, 2010.

LOPES, L. E.; BUZATO, S. Variation in pollinator assemblages in a fragmented landscape and its effects on reproductive stages of a self-incompatible treelet, Psychotria suterella (Rubiaceae).

Oecologia, v.154, n.1, p.305-314, 2007.

MONTEIRO, R. et al. Morfologia e distribuição espacial das formas heterostílicas de Psychotria barbiflora DC. (Rubiaceae). Naturalia, v.16, n.2, p.137-146, 1991.

MURCIA, C. Forest fragmentation and the pollination of neotropical plants. In: SCHELLAS, J.; GREENBERG, R. (Eds.). Forest patches in tropical landscapes. Washington: Island Press, 1996. p.19-36.

ORNELAS, J. F. et al. Reproductive ecology of distylous Palicourea padifolia (Rubiaceae) in a tropical montane cloud forest. I. Hummingbird's effectiveness as pollen vectors. American Journal of Botany, v.91, n.7, p.1052-1060, 2004a.
ORNELAS, J. F. et al. Reproductive biology of distylous Palicourea padifolia (Rubiaceae) in a tropical montane cloud forest.II Attracting and rewarding mutualistic and antagonistic visitors. American Journal of Botany, v.91, n.7, p.1061-1069, 2004b.

PAILLER, T.; THOMPSON, J. D. Distyly and variation in heteromorphic incompatibility in Gaertnera vaginata (Rubiaceae) endemic to La Reunion Island. American Journal of Botany, v.84, n3, p.315-327, 1997.

PEREIRA, Z. V.; CARVALHO-OKANO, R. M.; GARCIA, F. C. P. Rubiaceae Juss. da Reserva Florestal Mata do Paraíso, Viçosa, Minas Gerais, Brasil. Acta Botânica Brasilica, v.20, n.2, 207-224, 2006a.

PEREIRA, Z. V.; VIEIRA, M. F.; CARVALHOOKANO, R. M. Fenologia de floração, morfologia floral e sistema de incompatibilidade em espécies distílicas de Rubiaceae em fragmento florestal do sudeste brasileiro. Revista Brasileira de Botânica, v.29, n.3, p.471-480, 2006b.

RADFORD, A. E. et al. Vascular plant systematics. New York: Harper \& Row, 1974. 891p.

RAMOS, F. N.; SANTOS, F. A. M. Floral visitors and pollination of Psychotria tenuinervis (Rubiaceae): Distance from the anthropogenic and natural edges of an Atlantic Forest fragment. Biotropica, v.38, n.3, p.383-389, 2006.

RIBON, R. Demarcação de uma grade de trilhas no centro de pesquisa Mata do Paraíso, Viçosa, Minas Gerais. Revista Árvore, v.29, n.1, p.151-158, 2005.

RICHARDS, A. J. Plant breeding systems. London, Chapman \& Hall, 1997. 529p.

RIZZINI, C. T. Tratado de fitogeografia do Brasil: aspectos ecológicos, sociológicos e florísticos. São Paulo: Âmbito Cultural, 1992. 747p.

ROSSI, A. A. B.; OLIVEIRA, L. O.; VIEIRA, M. F. Distyly and variation in floral traits in natural populations of Psychotria ipecacuanha (Brot.) Stckes (Rubiaeae). Revista Brasileira do Botânica, v.28, n.2, p.285-294, 2005. 
SIH, A.; BALTUS, M. S. Patch size, pollinator behavior, and pollination limitation in catnip. Ecology, v.68, n.6, p.1679-1690, 1987.

SILVA, C. A. Biologia reprodutiva de três species distílicas de Psychotria L. e efeitos da fragmentação florestal no sucesso reprodutivo e na diversidade genetic de P. hastisepala Müll.Arg. (Rubiaceae). 2007. 63f. Tese (Doutorado em Botânica) - Universidade Federal de Viçosa, Viçosa, MG, 2007.

SILVA, C. A.; VIEIRA, M. F.; AMARAL, C. H. Floral attributes, ornithophily and reproductive success of Palicourea longepedunculata (Rubiaceae), a distylous shrub in southeastern Brazil. Revista Brasileira de Botânica, v.33, n.2, p.207-213, 2010.

SOBREVILLA, C. Reproductive biology of Palicourea fendleri e P. petiolaris (Rubiaceae), heterostylous shrubs of a tropical cloud. Biotropica, v.15, n.3, p.161-169, 1983.

STATSOFT. Statistica for Windows (Computer Program Manual). Tulsa: 2000.

STONE, J. L. Components of pollination effectiveness in Psychotria suerrensis, a tropical distylous shrub. Oecologia, v.107, n.4, p.505-512, 1996.

TAYLOR, C. M. Conspectus of the genus Palicourea (Rubiaceae: Psychotrieae) with the description of some new species from Ecuador and Colombia. Annals of the Missouri Botanical Garden, v.84, n.2, p.224-262, 1996.
TEIXEIRA, L. A. G.; MACHADO, I. C. Biologia da polinização e sistema reprodutivo de Psychotria barbiflora DC. (Rubiaceae). Acta Botânica Brasílica, v.18, n.4, p.853-862, 2004a.

TEIXEIRA, L. A. G.; MACHADO, I. C. Sabicea cinera Aubl. (Rubiaceae): distilia e polinização em um fragmento de Floresta Atlântica em Pernambuco, Nordeste do Brasil. Revista Brasileira de Botânica, v.27, n.1, p.193-204, 2004 b.

TOMIMATSU, H.; OHARA, M. Effects of forest fragmentation on seed production of the understory herb Trillium camschatcense.

Conservation Biology, v.16, n.5, p.12771285, 2002.

VELOSO, H. P.; RANGEL-FILHO, A. L. R.; LIMA, J. C. A. Classificação da vegetação brasileira, adaptada a um sistema universal. Rio de Janeiro: IBGE. Departamento de Recursos Naturais e Estudos Ambientais, 1991. 124p.

WEBB, C. J.; LLOYD, D. G. The avoidance of interference between the presentation of pollen and stigmas in angiosperms. II. Hercogamy. New Zealand Journal of Botany v.24, n.1, p.163-178, 1986.

ZAR, J. H. Bioestatistical analysis. 4.ed. New Jersey: Princt-Hall, 1999. 929p. 